\title{
Temperature dependence of the threshold electric field in a hot electron VCSEL
}

\author{
A. Erol, N. Balkan, M.Ç. Arıkan, A. Serpengüzel and J. Roberts
}

\begin{abstract}
The operation of the hot electron light emitting and lasing in semiconductor heterostructure - vertical cavity surface emitting laser (HELLISH-VCSEL) devices is based on hot carrier transport parallel to the layers of $\mathrm{Ga}_{1-x} \mathrm{Al}_{\mathrm{x}} A \mathrm{~s} \mathrm{p}-\mathrm{n}$ junction. It is therefore a field-effect device and the light emission from the device is independent of the polarity of the applied voltage. The authors present a study of the temperature dependence of the operational characteristics of the device. Experimental studies comprise the measurements of the I-V characteristics, electroluminescence, reflectivity, and temperature-dependent light-applied electric field (L-F) characteristics. To obtain the optimum operation temperature, the gain calculations, reflectivity spectra, and band gap calculations were carried out at different temperatures. The temperature dependence of the threshold electric field is compared with the model calculations, where radiative transitions without momentum $(k)$ selection are considered.
\end{abstract}

\section{Introduction}

HELLISH-VCSEL is a hot electron surface-emitting laser operating at room temperature and consisting of a HELLISH structure as the active layer of the microcavity. HELLISH is a longitudinal electron transport structure comprising a GaAs quantum well (QW) located on the $n$ side of the depletion layer of the $\mathrm{Ga}_{1-x} \mathrm{Al}_{x} \mathrm{As} \mathrm{p}-\mathrm{n}$ junction. Details of operation of the HELLISH can be found elsewhere [1-5]. Briefly, the electric field applied along the layers provides heating of the electrons and holes in their respective channels in the $\mathrm{Ga}_{1-x} \mathrm{Al}_{x} \mathrm{As} \mathrm{p}-\mathrm{n}$ junction as illustrated in Fig. 1. As the applied electric field is increased, electrons and holes in their respective channels become hot. (At a given value of the electric field, electrons, which have smaller effective masses and higher mobilities, are excited to higher energy levels than holes. Therefore, they have a higher non-equilibrium temperature than hole.) Hot electrons are injected by mainly tunnelling into the QW, resulting in the accumulation of negative charge in the depletion layer [1-5]. To preserve charge neutrality, the device self-biases itself reducing the potential barrier for the holes, and hence excess holes also move into the QW by diffusion, where they recombine with the electrons.

In the work presented here, we report on the experimental and theoretical studies of the temperature-dependent

\section{(C) IEE, 2003}

IEE Proceedings online no. 20030433

doi: 10.1049/ip-opt:20030433

Paper first received 7th October 2002 and in revised form 4th March 2003 A. Erol and M.Ç. Arıkan are with the Department of Physics, Istanbul University, Vezneciler, Istanbul 34459, Turkey

N. Balkan is with the Department of Electronics Systems Engineering, Essex University, Colchester CO4 3SQ, UK

A. Serpengüzel is with the Department of Physics, Koç University, Rumeli Feneri Yolu, Sariyer, Istanbul 34450, Turkey

J. Roberts is with the Department of Electronics and Electrical Engineering, Sheffield University, Sheffield SI 3JD, UK operation of the device, which is designed to operate as a vertical cavity surface-emitting laser.

\section{Experimental results and discussion}

Figure 2 shows the schematic diagram of the device grown by metalorganic vapour phase epitaxy (MOVPE) on a semiinsulating GaAs substrate. HELLISH forms the cavity of the HELLISH-VCSEL and is placed between the upper and lower distributed Bragg reflectors (DBRs). The lower DBR contains 27 periods of undoped $\mathrm{Ga}_{1-x} \mathrm{Al}_{x} \mathrm{As} / \mathrm{AlAs}$ layers, providing a reflectivity better than $99 \%$, while the upper DBR has 17 periods of similar layers, in order to allow output coupling via the sample surface.

HELLISH-VCSEL differs from the conventional VCSELs with its carrier injection mechanism, and hence the configuration of the electrical contacts. The conventional VCSELs utilise ring contacts on the top surface. In such a configuration the current is injected through the DBRs, and light emission is from the centre of the ring contact. This can be a drawback in the VCSEL operation, because of the finite resistivity of the DBR layers, leading to the excessive Joule heating and consequently to the undesired temperature shift of the cavity resonance and the gain peak. In the HELLISH-VCSEL, however, the two point contacts are diffused through all the layers; therefore, the current is injected into the active layer without passing through the DBRs as shown in Figs. $2 a$ and $2 b$.

The $\mathrm{Au}-\mathrm{Ge}-\mathrm{Ni}$ contacts are evaporated and then diffused through the layers as shown in Fig. $2 a$. Both the n- and p-layers of the active region are heavily doped. Therefore, the contacts are believed to be ohmic to all the layers at the typical operation temperatures between $77 \mathrm{~K}$ and room temperature $(300 \mathrm{~K})$. If the contacts were not ohmic, the light emission from the surface of the device would not be uniform, but concentrated in the cathode region [6].

The device is operated in the pulse mode, where short voltage pulses with $1 \mu$ s duration were applied along the layers. The duty cycle was kept below $0.01 \%$ to avoid excessive Joule heating at high fields. Electroluminescence (EL) measurements were performed in the temperature 


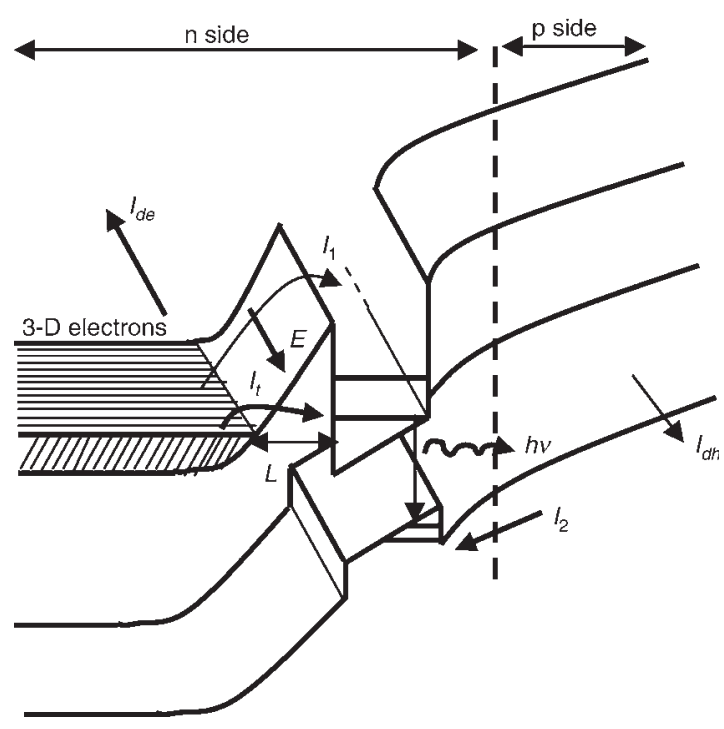

Fig. 1 Schematic diagram of HELLISH device operation

$E$ is the applied electric field; $I_{1}$ and $I_{\mathrm{t}}$ are injection of excess electrons by thermionic emission and tunnelling, respectively. $I_{\mathrm{de}}$ and $I_{\mathrm{dh}}$ are the electron and hole drift currents, respectively, and $I_{2}$ is the hot hole diffusion in the quantum well, after the self-biasing of the device as described in the text

range between $77 \mathrm{~K}$ and $300 \mathrm{~K}$. The light emission from the surface was collected, dispersed and measured with a $1 / 3 \mathrm{~m}$ monochromator and a cooled GaAs photomultiplier (PMT) assembly. The output signal from the PMT was detected using a boxcar averager. The room temperature emission is shown in Fig. 3. The spectrum peaks at $824 \mathrm{~nm}(1.50 \mathrm{eV})$, corresponding to the cavity resonance wavelength of the VCSEL. Measurements of emitted light intensity, integrated over the wavelength against the applied electric field were used to determine the threshold field at different temperatures as shown in Fig. 4. At low applied electric fields, the emission from the device is due to spontaneous emission. When the applied field is increased and more carriers are injected into the active layer, the gain in the device equals to the losses, and the device reaches the lasing threshold. At room temperature the threshold electric field is around $75 \mathrm{~V} / \mathrm{cm}$. The tendency towards saturation at $400 \mathrm{~V} / \mathrm{cm}$ is probably due to Joule heating of the device, when the gain peak moves further away from the cavity resonance due to the disparity in their temperature coefficients.

The threshold electric field, obtained from the integrated $\mathrm{L}-\mathrm{F}$ measurements, is plotted against temperature between $T=77 \mathrm{~K}$ and $300 \mathrm{~K}$ in Fig. 5. This curve exhibits a parabolic behaviour with a broad minimum at a temperature around $190 \mathrm{~K} \pm 20 \mathrm{~K}$. The parabolic behaviour can be understood in terms of the mismatch between the temperature dependences of the gain peak and the cavity resonance. Below $190 \mathrm{~K}$ the gain peak blue-shifts with respect to the cavity resonance, while above $180 \mathrm{~K}$ it redshifts with respect to the cavity resonance. Both gain peak and the cavity resonance coincide at $T \approx 190 \mathrm{~K}$. The temperature dependence of cavity resonance is determined by the temperature dependence of the refractive indices, while the gain spectrum follows the temperature dependence of the band gap energy. For GaAs $/ \mathrm{Ga}_{1-x} \mathrm{Al}_{x}$ As based devices gain spectrum shifts at a rate of $0.32-0.33 \mathrm{~nm}{ }^{\circ} \mathrm{C}^{-1}$ $[7,8]$, faster than that of the cavity resonance (0.06-0.09 $\left.\mathrm{nm}^{\circ} \mathrm{C}^{-1}[9,10]\right)$. At low temperatures the cavity resonance is situated on the low energy side of the gain spectrum, resulting in a higher threshold voltage or current. An increment in temperature leads to a better match
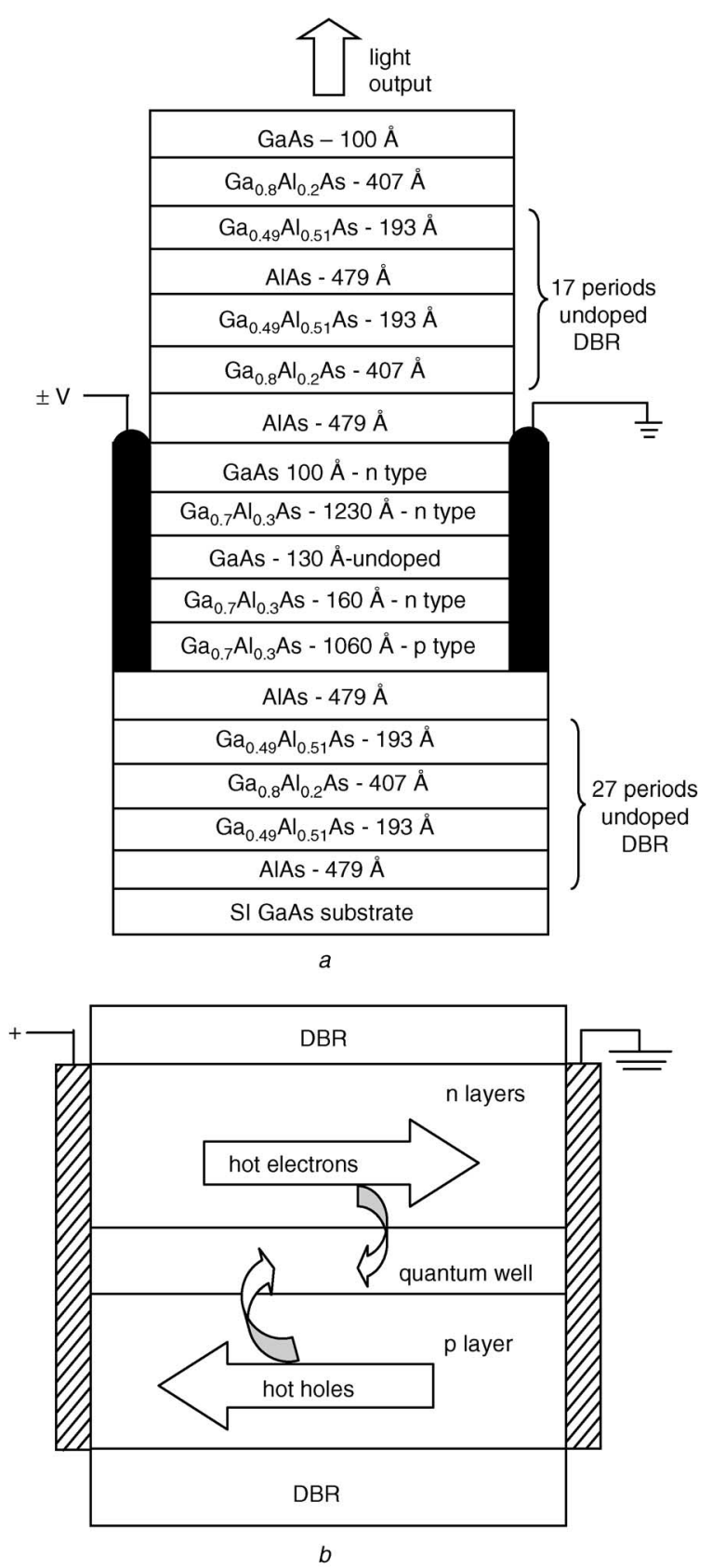

Fig. 2 Schematic diagram (a) and operation mechanism (b) of HELLISH-VCSEL

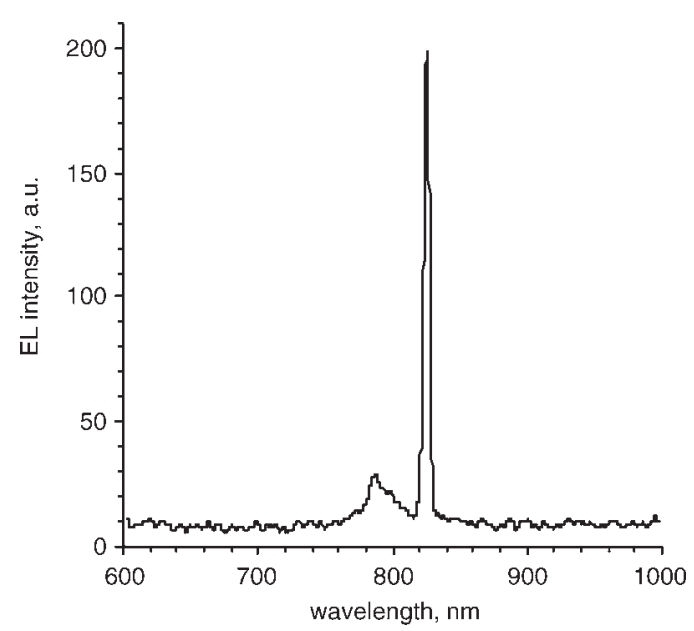

Fig. 3 EL spectrum at $T=300 \mathrm{~K}$ 


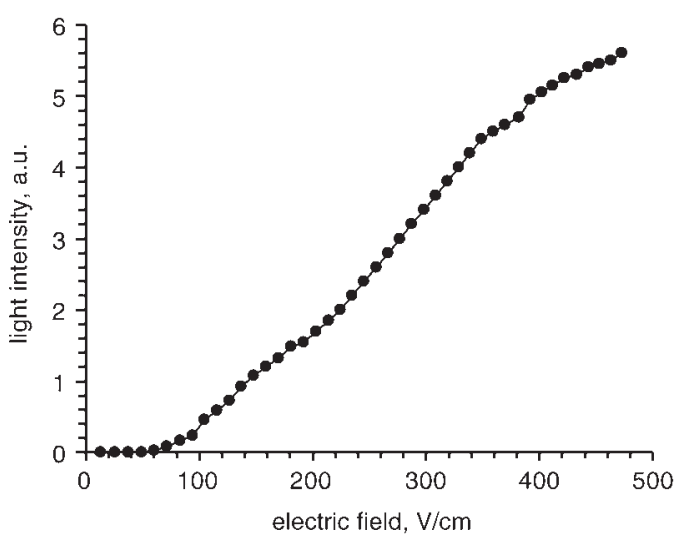

Fig. 4 Integrated $L-F$ measurement at $T=300 \mathrm{~K}$

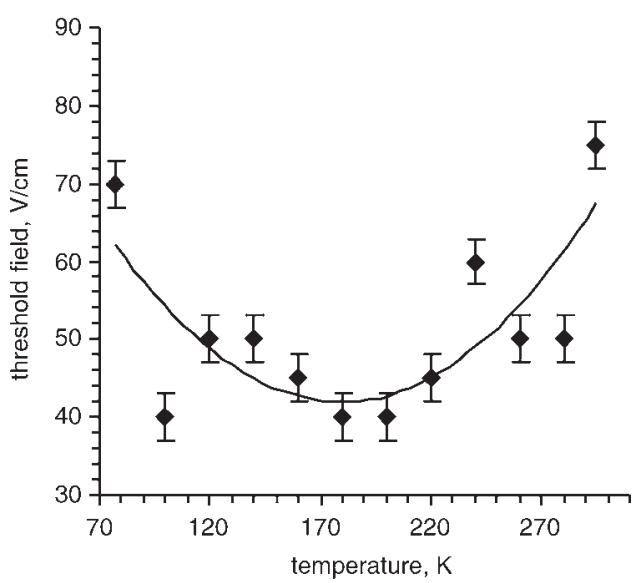

Fig. 5 Temperature dependence of threshold electric field

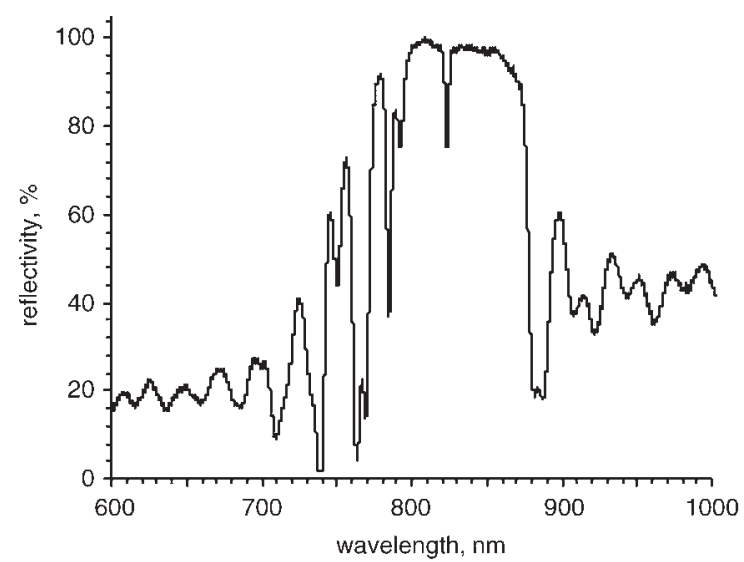

Fig. 6 Reflectivity spectrum HELLISH-VCSEL at T =300 K

to the cavity resonance and the gain peak, hence the threshold voltage decreases defining the observed minimum. As the temperature is increased further, the relative positions of the cavity resonance and the gain peak move apart, and cavity resonance coincides with the high-energy tail of the gain spectrum. This results in the increased threshold voltage or current as observed [11-13].

The cavity resonance wavelength was determined using the reflectivity spectroscopy. The tunable light was provided by a $100 \mathrm{~W}$ tungsten lamp, dispersed by a single grating monochromator, and chopped at a
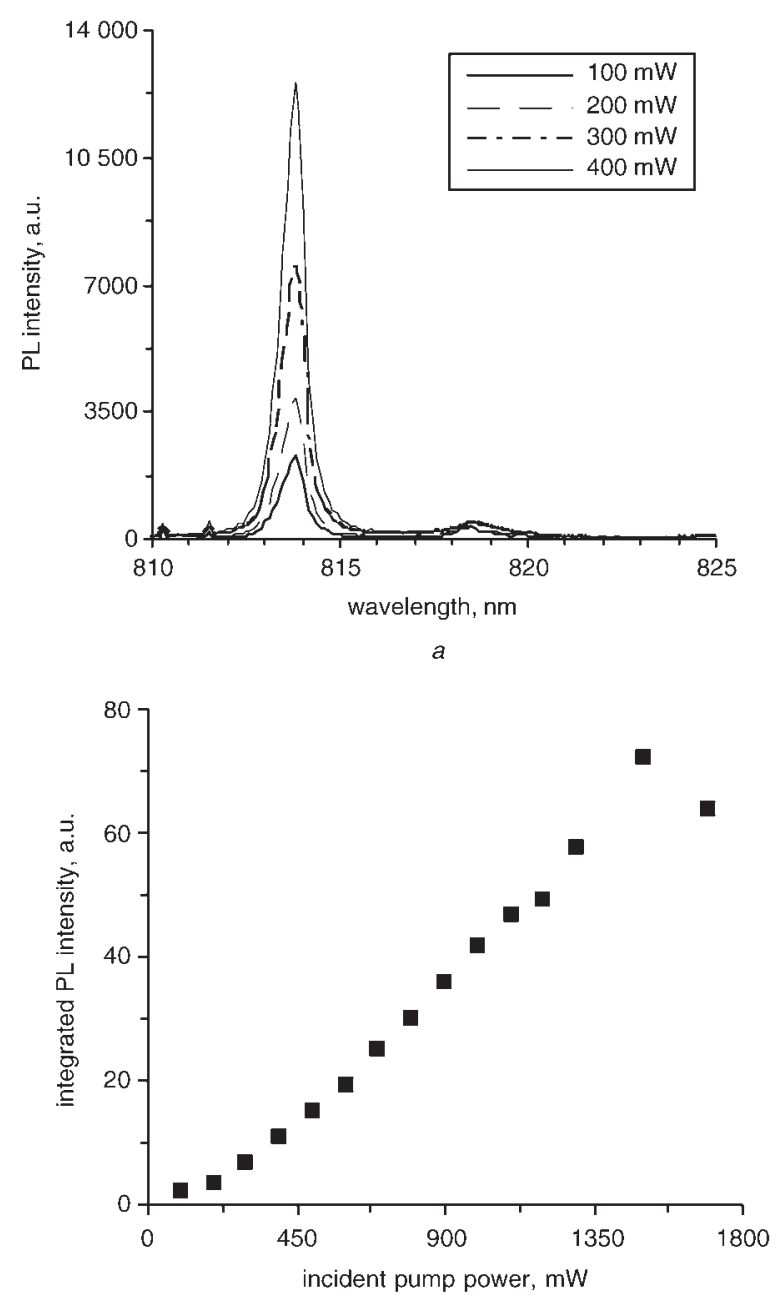

$b$

Fig. 7 Photoluminescence results

$a$ PL spectra at different incident pump powers

$b$ Integrated PL intensity against incident pump power at $T=19 \mathrm{~K}$

frequency of $185 \mathrm{~Hz}$. The reflected light from the sample surface was focused onto a silicon detector, and the detector output was measured using a lock-in amplifier. The reflectivity measurement was performed at normal incidence at room temperature. The resolution of the setup was $0.5 \mathrm{~nm}$. Figure 6 shows the reflectivity spectrum of the HELLISH-VCSEL. It is clear from the spectrum in Fig. 6 that the cavity resonance is located at $824 \mathrm{~nm}$, which is in good agreement with the EL emission in Fig. 3. The high reflectivity stop band is located between $800 \mathrm{~nm}$ and $870 \mathrm{~nm}$.

Photoluminescence measurements were also carried out as a function of pump intensity at temperatures between $19 \mathrm{~K}$ and $170 \mathrm{~K}$. Above $170 \mathrm{~K}$ no lasing was observed due to the available pump power being lower than the required lasing threshold at these temperatures. In the experiments the $514.5 \mathrm{~nm}$ line of a CW argon-ion laser, focused on to the device with a spherical lens, was used as the pump beam. Emission spectra measured at different pump powers are shown in Fig. 7a. It is clear from the Figure that the peak emitted light intensity increases and the linewidth decreases with increasing pump power. The PL intensity integrated over the wavelength against the incident pump power, measured at $T=19 \mathrm{~K}$ is shown in Fig. $7 b$. There is a welldefined optical power threshold of $\approx 300 \mathrm{~mW}$, above which the light intensity increases rapidly. There is a tendency towards saturation at higher pump powers. We believe this 
is also due to the heating of the device as observed commonly in conventional VCSEL structures as a result of the different temperature coefficients of the gain peak, and the cavity resonance as described in the previous Section. The optical power reaching the active layer of the device will be only a fraction of the incident power, due to the absorption and reflectivity in the upper layers.

\section{Theoretical modelling}

As described above, the performance of a VCSEL device depends strongly on the degree of overlap between the gain spectrum of the active region and the cavity resonance. If the cavity resonance wavelength coincides with peak wavelength of emission spectrum at an optimum operation temperature, the threshold will be at minimum. The ideal operation temperature for our laser is therefore $T=190 \pm 20 \mathrm{~K}$ as observed in Fig. 5 .

To calculate the operating temperature, we initially modelled the reflectivity spectrum using the transmission matrix theory [14]. For the $\mathrm{Ga}_{1-x} \mathrm{Al}_{x} \mathrm{As}$ material systems at photon energies below band gap, the wavelength dependence of the refractive index is given by Sellmeir equation at room temperature [15].

$$
n^{2}=A+\frac{B}{\lambda^{2}-C}-D \lambda^{2}
$$

where $\lambda$ is the free space wavelength in $\mu \mathrm{m}$ and $A, B, C$, and $D$ are Sellmeir coefficients, given in Table 1 . The reflectivity spectrum calculated at room temperature, using the refractive indices given in Table 1 and the growth parameters of Fig. $2 a$ are shown in Fig. 8. In the calculations, absorption is not taken into account. There is a sharp dip in the high reflectivity stop band at $830 \mathrm{~nm}$ $(1.49 \mathrm{eV})$, corresponding to the cavity resonance wavelength. This wavelength is higher than the experimentally measured value (Fig. 6) by $6 \mathrm{~nm}$. The small mismatch between the experimental and theoretical cavity resonance wavelength is probably due to the small fluctuations in the layer thicknesses of the device [16]. To obtain the best fit to the experimentally measured stop band and the cavity wavelength, we took the layer thickness as an adjustable parameter in the calculations. We obtain the best fit to the experimentally measured values for a layer thickness $0.9 \%$ lower than the nominal values of Fig. $2 a$. The spectrum calculated using the best fit values is also shown with dashed lines in Fig. 8. To determine the temperature dependence of the cavity resonance, the temperature dependence of the refractive index, which is given by the following equation, was used:

$$
n=n_{0}+\Delta n T
$$

Table 1: Sellmeir coefficients used for refractive index calculation in $\mathrm{Ga}_{1-x} \mathrm{Al}_{x} \mathrm{As}$

\begin{tabular}{lll}
\hline GaAs & & $\mathrm{Ga}_{1-x} \mathrm{Al}_{x} \mathrm{As}$ \\
\hline$A$ & 10.9060 & $10.9060-2.92 x$ \\
$B$ & 0.97501 & 0.97501 \\
$C$ & 0.27969 & $(0.52868-0.735 x) 2 ; x \leq 0.36$ \\
& $(0.30386-0.105 x) 2 ; x \geq 0.36$ \\
$D$ & 0.002467 & $0.002467(1.41 x+1)$ \\
$\left(n_{\mathrm{GaAs}}=3.501\right)$ & $\left(n_{\mathrm{AlAs}}=2.999\right)$ \\
\hline
\end{tabular}

where $M$ is the number of quantum wells, $B$ is the bimolecular recombination constant, $n$ is the refractive index, $L_{z}$ is the width of the QWs, $c$ is the speed of light, $\hbar$ is the Planck's constant, $k$ is Boltzmann's constant, $T$ is the temperature, and $m_{\mathrm{c}}$ and $m_{\mathrm{v}}$ are the electron and hole effective masses, respectively. $\Delta E$ is the difference between photon energy and the energy band gap $\left(E-E_{\mathrm{g}}\right), E_{\mathrm{fn}}$ the electron quasi-Fermi level measured from the conduction band edge, $E_{\mathrm{fp}}$ the hole Fermi level, measured from valence band edge. In this case the position of the quasi-Fermi levels are between the first and second sub-bands. The values of quasi-Fermi levels, measured from band edge, are obtained using charge neutrality condition, 

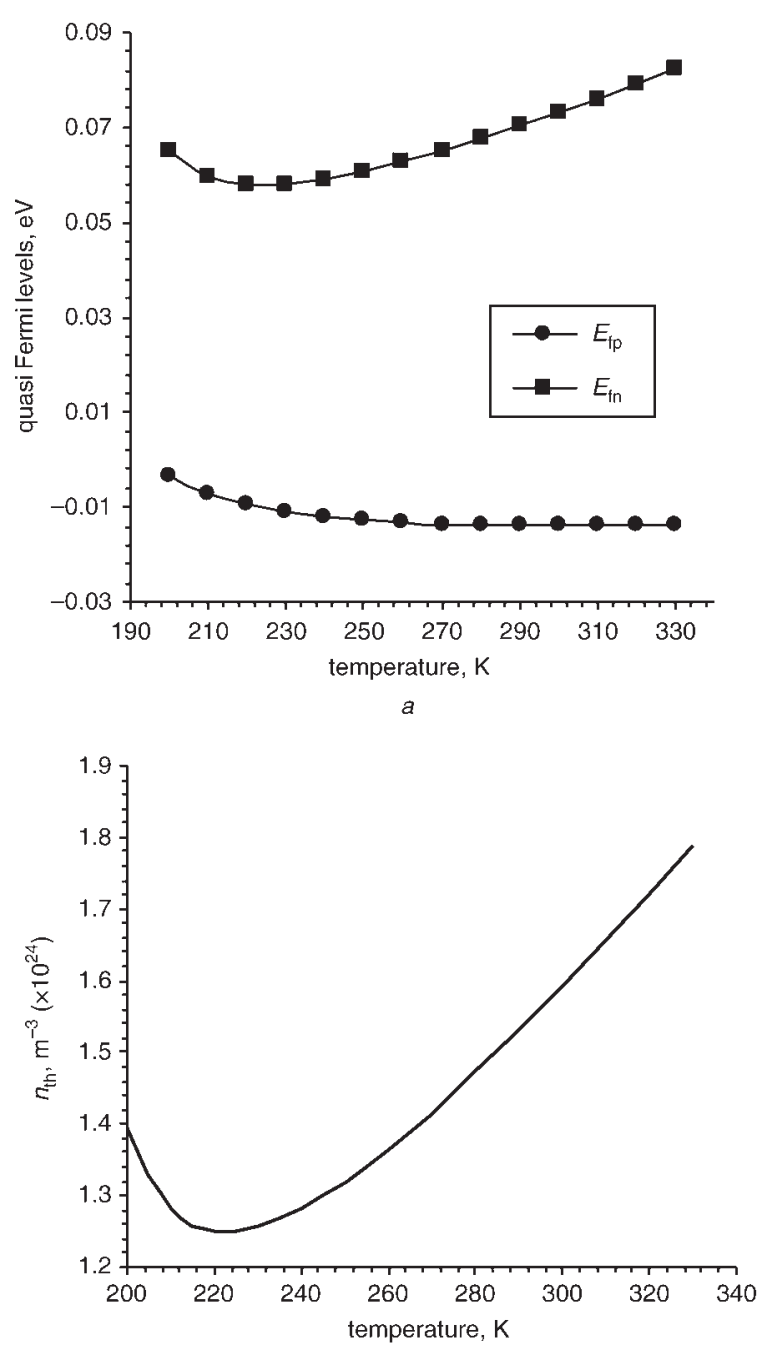

$b$

Fig.9 Quasi-Fermi levels and $n_{\text {th }}$ as functions of the temperature $a$ Quasi-Fermi levels

$b n_{\text {th }}$

$$
\begin{aligned}
& \left(1+\mathrm{e}^{E_{\mathrm{fn}} / k T}\right)\left(1+\mathrm{e}^{E_{\mathrm{fn}}-\Delta E_{\mathrm{n}}}\right) \\
& =\left(1+\mathrm{e}^{E_{\mathrm{fp}} / k T}\right)^{Z}\left(1+\mathrm{e}^{\left(E_{\mathrm{fp}}-\Delta E_{\mathrm{p}}\right) / k T}\right)^{Z}
\end{aligned}
$$

where $Z$ is the ratio of hole to electron mass $(Z=$ $\left.m_{\mathrm{v}} / m_{\mathrm{c}}\right), \Delta E_{\mathrm{n}}$ the difference between the first and second electron sub-bands, and $\Delta E_{\mathrm{p}}$ the energy difference between the first and second hole sub-bands. The corresponding carrier concentration is given by,

$n=p=\frac{m_{\mathrm{c}} k T}{\pi \hbar^{2} L_{z}} \ln \left(\left(1+\exp \left(\frac{E_{\mathrm{fn}}}{k T}\right)\right)\left(1+\exp \left(\frac{E_{\mathrm{fn}}-\Delta E_{\mathrm{n}}}{k T}\right)\right)\right)$

The effective band gap $E g_{\text {eff }}$ used in gain calculations is given by $E g_{\text {eff }}=E_{\mathrm{g}}+E_{1 \mathrm{n}}+E_{1 \mathrm{p}}$, where $E_{1 \mathrm{n}}$ and $E_{1 \mathrm{p}}$ are the first electron and hole sub-band energy, measured from conduction and valence band edges, respectively. To calculate the sub-band energies in respective bands, infinitive barrier approximation was used [25] and the temperature dependence of the energy band gap of GaAs was calculated using the Varshni equation [26],

$$
E_{\mathrm{g}}(T)=1.519-\frac{\left(5.405 \times 10^{-4}\right) T^{2}}{204+T}(\mathrm{eV})
$$

\begin{tabular}{|c|c|c|}
\hline Symbol & Description & Value \\
\hline$B$ & $\begin{array}{l}\text { bimolecular recombination } \\
\text { constant }\end{array}$ & $1.4 \times 10^{-10} \mathrm{~cm}^{3} / \mathrm{s}$ \\
\hline$\Delta E_{\mathrm{n}}$ & $\begin{array}{l}\text { difference between first } \\
\text { and second sub-band in } \\
\text { conduction band }\end{array}$ & $61.18 \mathrm{meV}$ \\
\hline$\Delta E_{\mathrm{p}}$ & $\begin{array}{l}\text { Difference between first } \\
\text { and second sub-band in } \\
\text { valence band }\end{array}$ & $14.25 \mathrm{meV}$ \\
\hline$E_{1 \mathrm{n}}$ & $\begin{array}{l}\text { energy of first electron } \\
\text { sub-band }\end{array}$ & $20.6 \mathrm{meV}$ \\
\hline$E_{1 \mathrm{p}}$ & energy of first hole sub-band & $4.76 \mathrm{meV}$ \\
\hline$L_{z}$ & width of quantum well & $130 \AA$ \\
\hline$N$ & refractive index & 3.6 \\
\hline$M$ & number of quantum wells & 1 \\
\hline$m_{\mathrm{c}}$ & effective electron mass & $0.0665 \mathrm{~m}$ \\
\hline$m_{\mathrm{p}}$ & effective hole mass & $0.34 \mathrm{~m}$ \\
\hline$M$ & free electron mass & $9.1 \times 10^{-31} \mathrm{~kg}$ \\
\hline$C$ & speed of light & $3 \times 10^{8} \mathrm{~m} / \mathrm{s}$ \\
\hline k & Boltzmann's constant & $8.617 \times 10^{-5} \mathrm{eV} / \mathrm{K}$ \\
\hline$\hbar$ & Planck's constant & $1.055 \times 10^{-34} \mathrm{~J} . \mathrm{s}$ \\
\hline
\end{tabular}

Table 2: Parameters used in the calculations of threshold properties of the HELLISH-VCSEL

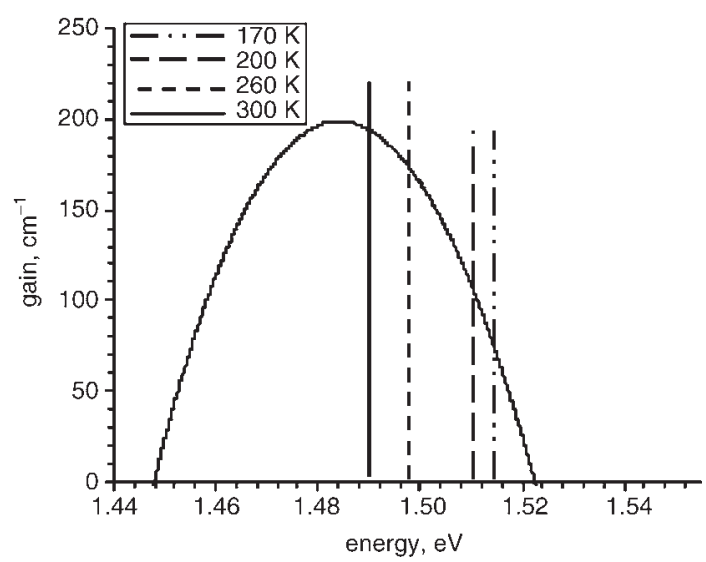

Fig. 10 Calculated gain spectra and photon energy of cavity resonance

The temperature dependence of the effective gap of the $130 \AA$ GaAs QW was calculated by adding the $E_{1 \mathrm{n}}$ and $E_{1 \mathrm{p}}$ energy values to the temperature dependence of bulk GaAs band gap, given in (6). The total optical loss in the cavity, including all internal and end losses, was assumed to be $100 \mathrm{~cm}^{-1}$. By setting the material gain equal to the loss (transparency condition) at a fixed temperature, temperature dependence of the quasi-Fermi levels are calculated from (3) and (4) as shown in Fig. 9a. The threshold carrier density $\left(n_{\text {th }}\right)$ shown in Fig. $9 b$ is calculated as a function of temperature using the values of quasi-Fermi levels in (5). The parameters used in the analysis are given in Table 2. It is clear from Fig. $9 b$ that the calculated threshold carrier density has a minimum at $220 \mathrm{~K}$. Gain spectrum is calculated as a function of temperature using (3) and the obtained temperature dependence of the photon energy of gain peak. This is shown in Fig. 10, where the gain spectra are plotted at several temperatures. Also shown in 


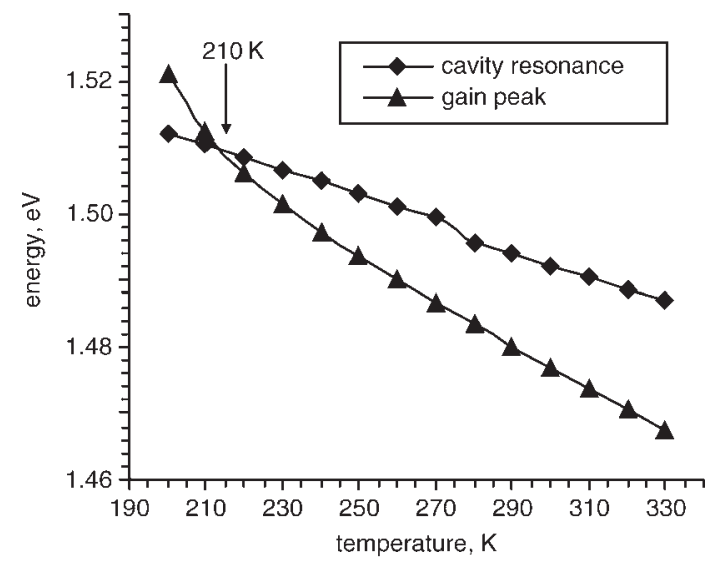

Fig. 11 Gain peak and cavity resonance as function of temperature

Fig. 10 are the corresponding cavity resonance energies calculated for the same temperatures. Finally, we have plotted the temperature dependences of both the cavity resonance and the gain peak in Fig. 11. It is evident from Figs. 10 and 11 that the two curves intersect at a temperature of $T \sim 210 \mathrm{~K}$, which is close to the observed temperature range for the broad minimum in the threshold voltage.

\section{Conclusions}

The temperature dependence of the threshold field for HELLISH-VCSEL is investigated. The experimental results are in agreement with the model calculations using the no- $k$ selection rule. The optimum operation temperature was obtained from the intersection of the temperaturedependent cavity resonance and the gain peak curves.

\section{Acknowledgments}

A. Erol is grateful to the British Council in Turkey and the Scientific and Technical Research Council of Turkey (TUBITAK) for their financial support during her stay at the University of Essex.

\section{References}

1 Straw, A., Balkan, N., O'Brien, A., Cunha, A.D., Gupta, R., and Arikan, M.C.: 'Hot electron light emitting and lasing semiconductor heterostructure: Type 1', Superlattices Microstruct., 1995, 18, pp. 33-43

2 O'Brien, A., Balkan, N., Boland-Thoms, A., Adams, M., Bek, A., Serpengüzel, A., Aydinli, A., and Roberts, J.: 'Super-radiant surface emission from a quasi-cavity hot electron light emitter', Opt. Quantum Electron., 1999, 31, pp. 183-190
3 O'Brien, A., Balkan, N., and Roberts, J.: 'Ultra bright surface emission from a distributed Bragg deflector hot electron light emitter', Appl. Phys. Lett., 1997, 70, pp. 366-368

4 Balkan, N., Serpengüzel, A., O'Brien-Davies, A., Sokmen, I., Hepburn, C., Potter, R., Adams, M.J., and Roberts, J.S.: 'VCSEL structure hot electron light emitter', Mater. Sci. Eng. B, Solid-State Mater. Adv. Technol., 2000, 74, pp. 96-100

5 Balkan, N., O'Brien, A., Boland-Thoms, A., Potter, R., Poolton, N., Adams, M.J., Masum, J., Bek, A., Serpengüzel, A., Aydinli, A., and Roberts, J.: 'The operation of a novel hot electron vertical cavity surface emitting laser', Proc. SPIE-Int. Soc. Opt. Eng., 1998, 3283, pp. $162-170$

6 Balkan, N.: 'Non-linear carrier dynamics in hot electron vertical cavity surface emitting laser', Physica B, 1999, 272, pp. 480-483

7 Van Der Ziel, J.P., Deppe, D.G., Chand, N., Zydzik, G.J., and Chun, S.G.: 'Characteristics of single- and two-dimensional phase coupled arrays of vertical cavity surface emitting GaAs-AlGaAs lasers', IEEE J. Quantum. Electron., 1990, 26, pp. 1873-1882

8 Scott, J.W., Corzine, S.W., Young, D.B., and Coldren, L.A.: 'Modelling the current to light characteristics of index guided vertical cavity surface emitting lasers', Appl. Phys. Lett., 1993, 62, pp. 1050-1052

9 Geels, R.S., Thibeault, B.J., Corzine, S.W., Scott, J.W., and Coldren, L.A.: 'Design and characterization of $\mathrm{In}_{0.2} \mathrm{Ga}_{08} \mathrm{As}$ MQW verticalcavity surface-emitting lasers', IEEE J. Quantum Electron., 1993, 29, pp. 2977-2987

10 Wipiejewski, T., Panzlaff, K., Zeeb, E., and Ebeling, K.J.: 'Tuneable extremely low-threshold vertical-cavity laser-diodes', IEEE Photonics Technol. Lett., 1993, 5, pp. 889-892

11 Nakwaski, W.: 'Thermal aspects of an efficient operation of vertical cavity surface emitting lasers', Opt. Quantum Electron., 1996, 28 pp. $335-352$

12 Tell, B., Brown-Goebeler, K.F., Leibenguth, R.E., Baez, F.M., and Lee, Y.H.: 'Temperature dependence of GaAs-AlGaAs vertical cavity surface emitting lasers', Appl. Phys. Lett., 1992, 60, pp. 683-685

13 Piprek, J., Akulova, Y.A., Coldren, L.A., and Bowers, J.E.: 'Minimum temperature sensitivity of $1.55 \mu \mathrm{m}$ vertical-cavity lasers at $30 \mathrm{~nm}$ gain offset', Appl. Phys. Lett., 1998, 72, pp. 1814-1817

14 Macleod, H.A.: 'Thin film optical filters' (Adam Hilger Ltd., Bristol, 1986, 2nd edn.)

15 Boyd, J.T.: 'Theory of parametric oscillation phase matched in GaAs thin film guides', IEEE J. Quantum Electron., 1972, 8, pp. 788-792

16 Sale, T.E.: 'Vertical cavity surface emitting lasers' (John Wiley, New York, 1995)

17 Casey, H.C., Jr., and Panish, M.B.: 'Heterostructure lasers, Part A fundamental principles' (Academic Press, New York, 1978)

18 Lasher, G., and Stern, F.: 'Spontaneous and stimulated recombination radiation in semiconductors', Phys. Rev., 1964, 133, pp. A553-A563

19 Marinelli, F.: 'Approximate calculation of the spectral function for the stimulated recombination radiation in semiconductors', Solid-State Electron., 1965, 8, pp. 939-942

20 Taylor, G.W.: 'Theory of operation of the quantum well injection laser without $k$ selection', J. Appl. Phys., 1991, 70, pp. 2508-2535

21 Taylor, G.W., and Evaldsson, P.A.: 'Temperature dependent operation of the vertical cavity surface emitting laser', IEEE J. Quantum Electron., 1994, 30, pp. 2262-2278

22 Masum, J., Balkan, N., and Adams, M.J.: 'Temperature dependence of $1.55 \mu \mathrm{m}$ VCSELs', Opt. Commun., 1998, 154, pp. 43-46

23 Masum, J., Ramoo, D., Balkan, N., and Adams, M.J.: 'Temperature dependence of the spontaneous emission factor in VCSELs', IEE Proc., Optoelectron., 1999, 146, pp. 245-251

24 Sceats, R., Ramoo, D., Masum, J., Balkan, N., Adams, M.J., Dann, A.J., Perrin, S.D., Reid, I., Reed, J., Cannard, P., Fisher, M.A., Elton, D.J., and Harlow, M.: 'GaInAsP/InP vertical cavity surface emitting laser for $1.55 \mu \mathrm{m}$ operation', Proc. SPIE-Int. Soc. Opt. Eng., 1999, 3625, pp. $315-323$

25 Chuang, S.L.: 'Physics of optoelectronic devices' (John Wiley, New York, 1995)

26 Varshni, Y.P.: 'Temperature dependence of the energy gap in semiconductors', Physica, 1967, 34, pp. 149-154

27 Joyce, W.B., and Dixon, R.W.: 'Electrical characterization of heterostructure lasers', J. Appl. Phys., 1978, 49, pp. 3719-3728 